ANNALES

POLONICI MATHEMATICI

$90.3(2007)$

\title{
Toric Hermitian surfaces and almost Kähler structures
}

\author{
by WŁodZimierz JeLONEK (Kraków)
}

\begin{abstract}
The aim of this paper is to investigate the class of compact Hermitian surfaces $(M, g, J)$ admitting an action of the 2-torus $T^{2}$ by holomorphic isometries. We prove that if $b_{1}(M)$ is even and $(M, g, J)$ is locally conformally Kähler and $\chi(M) \neq 0$ then there exists an open and dense subset $U \subset M$ such that $\left(U, g_{\mid U}\right)$ is conformally equivalent to a 4-manifold which is almost Kähler in both orientations. We also prove that the class of Calabi Ricci flat Kähler metrics related with the real Monge-Ampère equation is a subclass of the class of Gibbons-Hawking Ricci flat self-dual metrics.
\end{abstract}

0. Introduction. Let $(M, g, J)$ be a compact Hermitian surface with $J$-invariant Ricci tensor and even first Betti number $b_{1}$. Then it admits a non-trivial isometry group $G=\operatorname{Iso}(M, g)$. The identity component $G_{0}$ of $G$ is a compact, connected Lie group whose center is at least one dimensional. Let us assume that $\operatorname{dim} G \geq 2$. Then $G_{0}$ contains a 2 -torus $T^{2}$, which is contained in a maximal torus $T$ of $G_{0}$.

The aim of this paper is to investigate compact Hermitian surfaces $(M, g, J)$ admitting an action of a 2-torus $T^{2}$ by biholomorphic isometries. In $[\mathrm{L}]$ LeBrun has proved that if there exists a compact Einstein Hermitian structure different from the Page Einstein structure $[\mathrm{P}]$ on the first Hirzebruch surface $F_{1}$ then it is the blow up $M$ of $\mathbb{C} P^{2}$ in two or three points. It is known that the blow up of $\mathbb{C} P^{2}$ in three points admits an Einstein-Kähler metric [T-2]. On the other hand, the connected component of the group of isometries of $M$ in the case of the blow up of $\mathbb{C} P^{2}$ in two points is a 2 -torus $T^{2}$ which acts on $M$ by biholomorphic isometries. In [J-4] the author classified the Riemannian bi-Hermitian Gray structures (i.e. Gray metrics admitting two differently oriented orthogonal complex structures) on Hirzebruch surfaces which are of co-homogeneity 1 . (We should warn the reader that the notion of a bi-Hermitian surface has been recently used also in a different sense, namely to mean a surface admitting two positively oriented Hermitian structures). Other such surfaces if exist are ruled surfaces of genus 0 with

2000 Mathematics Subject Classification: 53C15, 53C55.

Key words and phrases: Hermitian surfaces, toric action, Kähler structure. 
an appropriate metric whose identity component of the isometry group is again a 2-torus $T^{2}$ acting by biholomorphic isometries. These two examples gave the motivation for the study of toric Hermitian surfaces.

1. Hermitian 4-manifolds. Let $(M, g, J)$ be an almost Hermitian manifold. We say that $(M, g, J)$ is a Hermitian manifold if its almost Hermitian structure $J$ is integrable. In the following we shall consider 4-dimensional Hermitian manifolds $(M, g, J)$ which we shall also call Hermitian surfaces. Such manifolds are always oriented and we choose an orientation in such a way that the Kähler form $\Omega(X, Y)=g(J X, Y)$ is self-dual (i.e. $\left.\Omega \in \bigwedge^{+} M\right)$. The vector bundle of self-dual forms admits a decomposition

$$
\bigwedge^{+} M=\mathbb{R} \Omega \oplus L M
$$

where $L M$ denotes the bundle of real $J$-skew-invariant 2 -forms (i.e. $L M=$ $\{\Phi \in \bigwedge M: \Phi(J X, J Y)=-\Phi(X, Y)\})$. The bundle $L M$ is a complex line bundle over $M$ with the complex structure $\mathcal{J}$ defined by $(\mathcal{J} \Phi)(X, Y)=$ $-\Phi(J X, Y)$. For a 4-dimensional Hermitian manifold the covariant derivative of the Kähler form $\Omega$ is locally expressed by

$$
\nabla \Omega=a \otimes \Phi+\mathcal{J} a \otimes \mathcal{J} \Phi
$$

where $\mathcal{J} a(X)=-a(J X)$. The Lee form $\theta$ of $(M, g, J)$ is defined by the equality

$$
d \Omega=\theta \wedge \Omega
$$

We have $\theta=-\delta \Omega \circ J$. The conformal scalar curvature $\kappa$ of an almost Hermitian manifold $(M, g, J)$ is defined by

$$
\kappa=\tau-\frac{3}{2}\left(|\theta|^{2}+2 \delta \theta\right) .
$$

We denote by $\mathfrak{i s o}(M)$ the Lie algebra of the group of isometries $\operatorname{Iso}(M)$ of $(M, g)$.

Proposition 1. Assume that $(M, g, J)$ is a compact locally conformally Kähler surface such that the Euler characteristic $\chi(M)$ is not zero or which is conformally Kähler. Then

$$
\theta(\xi)=0
$$

for every holomorphic Killing vector field $\xi \in \mathfrak{i s o}(M)$.

Proof. Note that $L_{\xi} \Omega=0$ and consequently $0=d L_{\xi} \Omega=L_{\xi} d \Omega=$ $L_{\xi}(\theta \wedge \Omega)=L_{\xi} \theta \wedge \Omega$. Consequently, $d \theta(\xi)=0$ and $\theta(\xi)=$ const. Note that $\theta^{\sharp} \in \mathfrak{X}(M)$ vanishes somewhere on $M$, since $\chi(M) \neq 0$ or because $\theta=d f$ and $M$ is compact. Consequently, $\theta(\xi)=0$.

Corollary 1. Let $(M, g, J)$ be a compact Hermitian locally conformally Kähler surface with $b_{1}$ even. Then an infinitesimal isometry $\xi$ of $(M, g)$ is holomorphic if and only if $\theta(\xi)=0$. 
Proof. The surface $(M, g, J)$ is conformally Kähler by a result of I. Vaisman [V]. Let $\theta=d f$ be the Lee form of $(M, g, J)$. Then $\bar{g}=\exp (-f) g$ is a Kähler metric on $M$. If $\theta(\xi)=0$ then $\xi f=0$ and consequently, $\xi \in \mathfrak{i s o}(M, \bar{g})$. On the other hand, it is well known that on a compact Kähler manifold every Killing vector field is holomorphic (see [Kob, p. 93, Th. 4.3]).

Let $R$ mean the curvature tensor of $(M, g)$ and let $\mathcal{R}$ denote the corresponding endomorphism $\mathcal{R}: \bigwedge^{2} M \rightarrow \bigwedge^{2} M$. Let us define $B=\frac{1}{2}(\mathcal{R}-* \mathcal{R} *)$; $W=\frac{1}{2}(\mathcal{R}+* \mathcal{R} *)_{0}=\frac{1}{2}(\mathcal{R}+* \mathcal{R} *)-\frac{\tau}{12} \mathrm{Id} ; W^{+}=\frac{1}{2}(W+* W) ; W^{-}=$ $\frac{1}{2}(W-* W)$. Then

$$
\mathcal{R}=\frac{\tau}{12} \mathrm{Id}+B+W^{+}+W^{-} .
$$

The Ricci tensor $\varrho$ of a Hermitian manifold $(M, g, J)$ is said to be Hermitian if $\varrho(X, Y)=\varrho(J X, J Y)$ for all $X, Y \in \mathfrak{X}(M)$. If $(M, g, J)$ is a Hermitian conformally Kähler surface then

$$
W^{+} \Omega=\frac{1}{6} \kappa \Omega, \quad W^{+} \Phi=-\frac{1}{12} \kappa \Phi \quad \text { if } \Phi \in L M .
$$

An opposite (almost) Hermitian structure on a Hermitian 4-manifold $(M, g, J)$ is an (almost) Hermitian structure $\bar{J}$ whose Kähler form (with respect to $g$ ) is anti-self-dual. A bi-Hermitian surface is a Hermitian surface which admits an opposite Hermitian structure.

On any almost Kähler non-Kähler 4-manifold $(M, g, J)$ there are two natural distributions $\mathcal{D}=\left\{X \in T M: \nabla_{X} J=0\right\}$ and $\mathcal{D}^{\perp}$ defined in the open set $U=\left\{x:\left|\nabla J_{x}\right| \neq 0\right\}$. We call $\mathcal{D}$ the nullity distribution of $(M, g, J)$. From $(1.2)$ it is clear that $\mathcal{D}$ is $J$-invariant and that $\operatorname{dim} \mathcal{D}=2$ in $U=\left\{x \in M: \nabla J_{x} \neq 0\right\}$. By $\mathcal{D}^{\perp}$ we denote the orthogonal complement of $\mathcal{D}$ in $U$. On $U$ we can define an opposite almost Hermitian structure $\bar{J}$ by setting $\bar{J} X=J X$ if $X \in \mathcal{D}^{\perp}$ and $\bar{J} X=-J X$ if $X \in \mathcal{D}$; we shall call it the natural opposite almost Hermitian structure. We have (see [J-3])

Theorem 1. Assume that $(M, g, J)$ is a compact conformally Kähler Hermitian surface with $J$-invariant Ricci tensor and let $\left(M, g_{0}, J\right)$ be a Kähler surface in the conformal class $(M,[g], J)$. Then both $(M, g, J)$, $\left(M, g_{0}, J\right)$ admit a holomorphic Killing vector field $\xi$ with zeros such that $\nabla_{\xi} J=0$, where $\nabla$ is the Levi-Civita connection of $(M, g)$. Moreover $\xi=$ $J \bar{\nabla} u$ where $\bar{\nabla}$ is the Levi-Civita connection of $\left(M, g_{0}\right), u$ is a positive, smooth function on $M$ such that $g=u^{-2} g_{0}$, and

$$
\begin{aligned}
\nabla_{\xi_{0}} \xi_{0} & =-\nabla \alpha+\alpha J \xi_{0}, \quad g_{0}(\xi, \xi)=\alpha^{2}, \\
\theta(X) & =-2 \alpha g\left(J \xi_{0}, X\right),
\end{aligned}
$$

where $\xi_{0}=\frac{1}{\sqrt{g(\xi, \xi)}} \xi$ is defined in $U=\left\{x: \xi_{x} \neq 0\right\}, \alpha=\frac{1}{2 \sqrt{2}} \sqrt{g(\nabla J, \nabla J)}$. The Killing vector field $\xi$ belongs to the center $\mathfrak{z}(\mathfrak{i s o}(M))$ of $\mathfrak{i s o}(M)$. 
We say that a holomorphic Killing vector field $\eta$ on $(M, g, J)$ is potential with potential function $f$ if $\eta=J(\nabla f)$ where $\nabla f$ is the gradient of $f$ with respect to $g$.

Corollary 2. Assume that $(M, g, J)$ is a compact conformally Kähler non-Kähler Hermitian surface with J-invariant Ricci tensor and let $\eta$ be a potential holomorphic Killing vector field for $(M, g, J)$. Then $\eta=c \xi$ for some $c \in \mathbb{R}-\{0\}$.

Proof. Since $(M, g, J)$ is conformally Kähler, there exists a non-constant function $\phi \in C^{\infty}(M)$ such that $\theta=d \phi$. It follows that the metric $\bar{g}=$ $\exp (-\phi) g$ is Kähler. Denote by $\bar{\nabla}$ the Riemannian connection for $(M, \bar{g})$. Then

$$
\bar{\nabla}_{X} Y=\nabla_{X} Y-\frac{1}{2}(d \phi(X) Y+d \phi(Y) X-g(X, Y) \nabla \phi) .
$$

Now assume that $\eta=\nabla f$ for some $f \in C^{\infty}(M)$. Then $\eta$ is a vector field with zeros. Since $\theta(\eta)=0$ it follows that $\eta$ is also a holomorphic Killing vector field for $(M, \bar{g}, J)$. Consequently, there exists a function $h \in C^{\infty}(M)$ such that $\eta=J(\bar{\nabla} h)$. Note that $\nabla h=\exp (-\phi) \bar{\nabla} h$. Consequently, $\exp (\phi) d h=d f$ and $d \phi \wedge d h=0$. It follows that there exists a function $c \in C^{\infty}(M)$ such that $\nabla f=c \nabla \phi$. Denote by $K_{X} Y$ the difference tensor $K_{X} Y=\bar{\nabla}_{X} Y-\nabla_{X} Y$. Then $K_{\eta} X=-\frac{1}{2}(d \phi(X) \eta-g(\eta, X) \nabla \phi)$. Since $\eta=c J(\nabla \phi)$ it is not difficult to show that $\left[K_{\eta}, J\right]=0$ and consequently $\nabla_{\eta} J=0$. It follows that $\eta \in$ $\Gamma(\mathcal{D})$. Since $\xi \in \Gamma(\mathcal{D})$ and $\theta(\eta)=0$ it follows that $\xi, \eta$ are parallel and consequently, there exists a constant $c \in \mathbb{R}$ such that $\eta=c \xi$.

2. Toric Hermitian surfaces. In this section we shall give the description of compact Hermitian surfaces whose group of holomorphic isometries contains a 2-torus.

Lemma A. Assume that a Riemannian manifold $(M, g)$ admits two commuting Killing vector fields $\xi, \eta$. Then

$$
R(\xi, \eta) X=[T, K] X
$$

where $T=\nabla \xi, K=\nabla \eta$ and $[T, K]=T \circ K-K \circ T$.

Proof. Define $\psi(x)=g\left(\xi_{x}, \eta_{x}\right)$. Since $[\xi, \eta]=0$ it is clear that $X \psi=$ $g\left(\nabla_{X} \xi, \eta\right)+g\left(\xi, \nabla_{X} \eta\right)=-2 g\left(\nabla_{\xi} \eta, X\right)$. Consequently,

$$
T \eta=K \xi=-\frac{1}{2} \nabla \psi \text {. }
$$

The relations (2.1) imply

$$
R(X, \xi) \eta+T \circ K X=-\frac{1}{2} \nabla_{X} \nabla \psi, \quad R(X, \eta) \xi+K \circ T X=-\frac{1}{2} \nabla_{X} \nabla \psi .
$$

Thus $R(\xi, \eta) X=R(X, \eta) \xi-R(X, \xi) \eta=[T, K] X$. 
Definition. A toric Hermitian surface $(M, g, J)$ is a Hermitian surface which admits two linearly independent, commuting holomorphic Killing vector fields $\xi, \eta$ such that $\Omega(\xi, \eta)=0$.

Let $T$ be a connected subgroup of $\operatorname{Iso}(M, g)$ corresponding to the Abelian Lie algebra $\mathfrak{t}=\operatorname{span}\{\xi, \eta\} \subset \mathfrak{i s o}(M, g)$. By a volume function of the toric action of $T$ on $(M, g)$ we mean any function $V=g(\xi, \xi) g(\eta, \eta)-g(\xi, \eta)^{2}$, where $\xi, \eta$ is any basis of $\mathfrak{t}$. Note that $V$ is determined uniquely up to a constant factor. Note that we do not assume that $T$ is a torus.

REMARK. Recall that a symplectic 4-manifold is called toric if it admits a Hamiltonian action of a 2 -torus $T$ with an invariant moment map. Thus if $(M, g, J)$ is Kähler then our definition does not coincide with the usual one. However, it does if $T$ is a 2-torus and $M$ is simply connected and Kähler. We have:

Proposition 2. Assume that $(M, g, J)$ is a compact locally conformally Kähler Hermitian surface with two commuting holomorphic Killing vector fields $\xi, \eta$ and non-vanishing Euler characteristic. Then $\Omega(\xi, \eta)=0$ on the whole of $M$. If $(M, g, J)$ is conformally Kähler and $\chi(M)=0$ then either $\Omega(\xi, \eta)=0$ on the whole of $M$, or $\Omega(\xi, \eta) \neq 0$ on the whole of $M$ and the Lee form of $(M, g, J)$ equals $\theta=-d \ln |\Omega(\xi, \eta)|$.

Proof. Note that

$$
d(\Omega(\xi, \eta))=d\left(i_{\xi} i_{\eta} \Omega\right)=d \Omega(\xi, \eta, \cdot)=-\theta \Omega(\xi, \eta),
$$

since Proposition 1 implies $\theta(\xi)=\theta(\eta)=0$. Consequently, in the open subset $U=\{x \in M: \Omega(\xi, \eta) \neq 0\}$ we have $\theta=-d \ln |\Omega(\xi, \eta)|$. Since $\theta$ is a smooth 1-form on $M$ it follows that either $U=\emptyset$ or $\Omega(\xi, \eta) \neq 0$ on the whole of $M$.

Now we shall show

Corollary 3. Let g be a Gray bi-Hermitian metric (see [J-1]) on one of the Hirzebruch surfaces $F_{k}$. Then $\left(F_{k}, g\right)$ is a toric Hermitian surface with respect to both complex structures $J, \bar{J}$ on $F_{k}$.

Proof. Let $\xi, \bar{\xi}$ be potential Killing holomorphic fields for $\left(F_{k}, g, J\right)$ and $\left(F_{k}, g, \bar{J}\right)$ respectively. If $\xi \wedge \bar{\xi}=0$ then $\left(F_{k}, g\right)$ is of co-homogeneity 1 and is toric with respect to $J, \bar{J}$. Assume that $\xi \wedge \bar{\xi} \neq 0$. From [J-1] it follows that the Lee forms $\theta, \bar{\theta}$ satisfy an equation

$$
\theta+\bar{\theta}=d \ln |\lambda-\mu| .
$$

Thus $\theta(\bar{\xi})=d \ln |\lambda-\mu|(\bar{\xi})-\bar{\theta}(\bar{\xi})=0$. Analogously $\bar{\theta}(\xi)=0$. It follows from Corollary 1 that $\xi \in \mathfrak{h o l}\left(F_{k}, \bar{J}\right)$ and $\bar{\xi} \in \mathfrak{h o l}\left(F_{k}, J\right)$. Theorem 1 implies $[\xi, \bar{\xi}]=0$. Since $\chi\left(F_{k}\right) \neq 0$ the result is a consequence of Proposition 2 . 
Let $\mathcal{F}$ be an oriented distribution of constant dimension $k$. Then by the characteristic form of $\mathcal{F}$ we mean the form $\omega_{\mathcal{F}}=e_{1} \wedge \cdots \wedge e_{k}$ where $\left\{e_{1}, \ldots, e_{k}\right\}$ is any oriented, orthonormal basis of $\mathcal{F}$.

Lemma B. Let $(M, g, J)$ be a 4-dimensional Hermitian manifold. Let $\xi, \eta$ be two linearly independent commuting Killing vector fields, holomorphic with respect to $J$ and such that $\Omega(\xi, \eta)=0$, where $\Omega$ is the Kähler form of $(M, g, J)$. Let $U=\left\{x:\left\|\xi_{x} \wedge \eta_{x}\right\| \neq 0\right\} \subset M$ and consider the distribution $V=\operatorname{span}_{\mathbb{R}}\{\xi, \eta\}$ in $U$. Then $U$ is an open and dense subset of $M, V^{\perp}=J V$, $K V \subset V^{\perp}, T V \subset V^{\perp}$ where $T, K$ are as in Lemma A. What is more, $V, J V$ are integrable distributions, the form $\Phi=\omega_{J V}+\omega_{V}$, where $\omega_{J V}, \omega_{V}$ are the characteristic forms of the foliations $V, J V$ respectively, belongs to $L_{J} M$, and $J V$ is totally geodesic. The characteristic form $\omega_{J V}$ of the foliation $J V$ is closed. Moreover if $(M, g, J)$ is Kähler, or compact and conformally Kähler, then $K V^{\perp} \subset V, T V^{\perp} \subset V$, the form $\Phi=\omega_{J V}+\omega_{V}$ is an eigenform of the positive Weyl tensor $W^{+}$, and the form $\bar{\Phi}=\omega_{J V}-\omega_{V}$ is an eigenform of the negative Weyl tensor $W^{-}$.

Proof. Note first that $U$ is an open and dense subset of $M$. It is clear from the assumptions that $V^{\perp}=J V$. Define $\phi(x)=g(\xi, \xi)_{x}, \psi(x)=g(\xi, \eta)_{x}$, $\chi(x)=g(\eta, \eta)_{x}$. Then $T \xi=-\frac{1}{2} \nabla \phi, T \eta=K \xi=-\frac{1}{2} \nabla \psi, K \eta=-\frac{1}{2} \nabla \chi$. Since $\xi, \eta$ are perpendicular to $\nabla \phi, \nabla \psi, \nabla \chi$ it follows that $K V \subset V^{\perp}$ and $T V \subset V^{\perp}$. Note that $J$ is integrable and consequently $J \xi, J \eta$ are real holomorphic vector fields. Thus $[J \xi, J \eta]=0$ and it follows that $J V$ is integrable. Moreover $J V$ is totally geodesic, since it is the orthogonal complement of a distribution spanned by Killing vector fields. Since the orthogonal complement of $J V$ is the involutive distribution $V$ it follows that the characteristic form of $J V$ is closed.

Let $E_{1}, E_{2}$ be an orthonormal basis of $V$. Consequently, $E_{3}=J E_{1}, E_{4}=$ $J E_{2}$ is an orthonormal basis of $V^{\perp}$. We have $\Omega=E_{1} \wedge E_{3}+E_{2} \wedge E_{4}$. The form $\Phi=E_{1} \wedge E_{2}-E_{3} \wedge E_{4}$ belongs to $L_{J} M$ and the form $\bar{\Phi}=E_{1} \wedge E_{2}+E_{3} \wedge E_{4}$ belongs to $\wedge^{-} M$. Note that $\omega_{V}=E_{1} \wedge E_{2}$ and $\omega_{J V}=-E_{3} \wedge E_{4}$ are the characteristic forms of the foliations $V, J V$ respectively. Now, assume that $(M, g)$ is conformally Kähler, i.e. there exists a function $f \in C^{\infty}(M)$ for which the metric $\exp (-f) g$ is Kähler, and $\theta=d f$. Note that $K J V \subset V$ if and only if $g(K J \eta, J \xi)=0$.

We shall show that $g\left(\nabla_{J \eta} \eta, J \xi\right)=0$. Note that

$$
\begin{aligned}
g\left(\nabla_{J \eta} \eta, J \xi\right) & =g\left(\nabla_{\eta}(J \eta), J \xi\right)=g\left(\nabla_{\eta} J(\eta), J \xi\right)+g\left(J \nabla_{\eta} \eta, J \xi\right) \\
& =g\left(\nabla_{\eta} J(\eta), J \xi\right) .
\end{aligned}
$$

On the other hand,

$$
g\left(\nabla_{X} J(Y), Z\right)=\frac{3}{2}(d \Omega(X, J Y, J Z)-d \Omega(X, Y, Z))
$$


since $J$ is Hermitian. Consequently,

$$
g\left(\nabla_{\eta} J(\eta), J \xi\right)=\frac{3}{2}(-d \Omega(\eta, J \eta, \xi)-d \Omega(\eta, \eta, J \xi))=-\frac{3}{2} d \Omega(\eta, J \eta, \xi) .
$$

If $(M, g, J)$ is Kähler then the result is obvious. Now assume that $M$ is compact conformally Kähler. It is clear from Corollary 1 that $\theta(\xi)=\theta(\eta)=0$. Thus $\Omega(\xi, \eta)=0$ implies that $d \Omega(\eta, J \eta, \xi)=0$ and consequently, $g(K J \eta, J \xi)$ $=0$. Analogously one can prove that $T J V \subset V$. Let $E_{1}, E_{2}$ be an orthonormal basis of $V$. Write $c(x)=\left\|\xi_{x} \wedge \eta_{x}\right\|$. Then

$$
c R\left(E_{1} \wedge E_{2}\right) X= \pm R(\xi, \eta) X= \pm[T, K] X .
$$

Note that $[T, K]$ preserves both distributions $V, J V$. Consequently,

$$
R\left(E_{1}, E_{2}\right)=\alpha \Phi+\beta \bar{\Phi} .
$$

It follows that $\mathcal{R}\left(E_{1} \wedge E_{2}\right)=\alpha \Phi+\beta \bar{\Phi}$. Note that $E_{1} \wedge E_{2}=\frac{1}{2}(\Phi+\bar{\Phi})$. Thus $\mathcal{R}\left(\frac{1}{2}(\Phi+\bar{\Phi})\right)=\alpha \Phi+\beta \bar{\Phi}$. Since for every holomorphic Killing vector field $\zeta$ we have $\theta(\zeta)=0$ it follows that both $\xi, \eta$ are holomorphic Killing vector fields for the Kähler metric $\exp (-f) g$. Note further that $\bar{\nabla}_{\xi} J=$ $\bar{\nabla}_{\eta} J=0$ since $\bar{\nabla} J=0$, where $\bar{\nabla}$ is the Levi-Civita connection of the Kähler metric $\exp (-f) g$. Consequently, $[\bar{T}, J]=[\bar{K}, J]=0$ where we have set $\bar{T}=\bar{\nabla} \xi, \bar{K}=\bar{\nabla} \eta$. It follows that $J \circ[\bar{T}, \bar{K}]=[\bar{T}, \bar{K}] \circ J$ and consequently $\overline{\mathcal{R}}\left(\frac{1}{2}(\Phi+\bar{\Phi})\right)=\bar{\beta} \bar{\Phi}$, where $\overline{\mathcal{R}}$ is the curvature tensor of the Kähler manifold $(M, \exp (-f) g)$. Since $\exp (-f) g$ is a Kähler metric and $\Phi \in L_{J} M$ it is clear that $\overline{\mathcal{R}}(\Phi)=0$. Consequently, $\overline{\mathcal{R}}(\bar{\Phi})=2 \bar{\beta} \bar{\Phi}$, which means that $\bar{W}(\bar{\Phi})=$ $\bar{W}^{-}(\bar{\Phi})=\lambda \bar{\Phi}$. Since the Weyl tensor $W$ is a conformal invariant of the conformal manifold $(M,[g])$ it follows that $\Phi, \bar{\Phi}$ are eigenvectors of the Weyl tensors $W^{+}, W^{-}$of $(M, g)$ respectively.

We also have $W(\Phi)=W^{+}(\Phi)=-\frac{\kappa}{12} \Phi$ where $\kappa$ is the conformal scalar curvature of $(M, g, J)$. Note also that $\alpha=\frac{1}{24}(\tau-\kappa)$.

Now we shall give several interesting examples of almost Kähler structures on compact and non-complete 4-dimensional Riemannian manifolds. Namely we shall prove

Theorem 2. Assume that $(M, g, J)$ is Kähler or compact conformally Kähler 4-dimensional toric Hermitian surface. Let $v$ be a volume function of a toric action. Then there exists an open and dense subset $U \subset M$ and a function $f \in C^{\infty}(U)$ such that the Hermitian surface $\left(U, g_{*}\right)$, where $g_{*}=$ $\left.f g\right|_{U}$, satisfies the following conditions:

(a) $U=\{x \in M: v(x) \neq 0\}$,

(b) $f=1 / \sqrt{v}$,

(c) the eigenspace $L_{J} M$ of the Weyl tensor $W^{+}$of $\left(U, g_{*}\right)$ is spanned by two Kähler forms $\Phi, \Psi$ of two orthogonal almost Kähler structures compatible with the metric $g_{*}$, 
(d) the almost Hermitian structure $\bar{\Phi}$ which is the natural opposite structure with respect to $\Phi$ is globally defined on $U$ and $\bar{\Phi}$ is an almost Kähler structure on $\left(U, g_{*}\right)$,

(e) the form $\bar{\Phi}$ is an eigenvector of the negative Weyl tensor $W^{-}$.

Proof. We have two holomorphic Killing fields $\xi, \eta$ such that $[\xi, \eta]=0$. Recall that if $\xi, \eta$ is a basis of an Abelian subalgebra $\mathfrak{t} \subset \mathfrak{i s o}(M, g)$ then the volume function of the toric action is $v=g(\xi, \xi) g(\eta, \eta)-g(\xi, \eta)^{2}$. Then $v \in C^{\infty}(M)$ and let $U=\{x \in M: v(x) \neq 0\}$. It is clear that $U$ is an open and dense subset of $M$. On $U$ there is defined a frame $\xi, \eta, J \xi, J \eta$. Note that any two of the vector fields of the frame commute. Denote by $\theta_{\xi}, \theta_{\eta}, \theta_{J \xi}, \theta_{J \eta}$ the dual co-frame. Then $d \theta_{\xi}=d \theta_{\eta}=d \theta_{J \xi}=d \theta_{J \eta}=0$. Define $V=\operatorname{span}\{\xi, \eta\}$. Then $V^{\perp}=J V=\operatorname{span}\{J \xi, J \eta\}$.

Now we define an orthonormal frame on $U$ by

where

$$
E_{1}=\frac{1}{\sqrt{\phi}} \xi, \quad E_{2}=\frac{1}{\sqrt{g\left(\eta^{\perp}, \eta^{\perp}\right)}} \eta^{\perp}, \quad E_{3}=J E_{1}, \quad E_{4}=J E_{2},
$$

$$
\eta^{\perp}=\eta-\frac{g(\xi, \eta)}{g(\xi, \xi)} \xi \quad \text { and } \quad \phi=g(\xi, \xi) .
$$

Let $\left\{\theta_{1}, \theta_{2}, \theta_{3}, \theta_{4}\right\}$ be a dual orthonormal co-frame. Then

$$
\begin{aligned}
\theta_{\xi} & =\frac{1}{\sqrt{\phi}}\left(\theta_{1}-\cos (\xi, \eta) \theta_{2}\right), \quad \theta_{\eta}=\frac{1}{\sqrt{g\left(\eta^{\perp}, \eta^{\perp}\right)}} \theta_{2}, \\
\theta_{J \xi} & =\frac{1}{\sqrt{\phi}}\left(\theta_{3}-\cos (\xi, \eta) \theta_{4}\right), \quad \theta_{J \eta}=\frac{1}{\sqrt{g\left(\eta^{\perp}, \eta^{\perp}\right)}} \theta_{4} .
\end{aligned}
$$

Note that

$$
\theta_{\xi} \wedge \theta_{\eta}=\frac{1}{\sqrt{v}} \theta_{1} \wedge \theta_{2}, \quad \theta_{J \xi} \wedge \theta_{J \eta}=\frac{1}{\sqrt{v}} \theta_{3} \wedge \theta_{4}
$$

If we change conformally the metric $g$ to $g_{*}=f g$ and define an orthonormal frame $\left\{E_{1}^{f}, E_{2}^{f}, E_{3}^{f}, E_{4}^{f}\right\}$ and an orthonormal co-frame $\left\{\theta_{1}^{f}, \theta_{2}^{f}, \theta_{3}^{f}, \theta_{4}^{f}\right\}$ with respect to $g_{*}$ analogously to the above then $\theta_{i}^{f}=\sqrt{f} \theta_{i}$. Consequently, if we take $f=1 / \sqrt{v}$ then

$$
\theta_{\xi} \wedge \theta_{\eta}=\theta_{1}^{f} \wedge \theta_{2}^{f}, \quad \theta_{J \xi} \wedge \theta_{J \eta}=\theta_{3}^{f} \wedge \theta_{4}^{f}
$$

on $U$. In what follows we shall only consider the metric $g_{*}$ on $U$ and we shall write $\theta_{i}=\theta_{i}^{f}$. Let $\Phi=\theta_{1} \wedge \theta_{2}+\theta_{3} \wedge \theta_{4}$. Note that the Kähler form $\Omega_{*}=$ $g_{*}(J X, Y)$ of the Hermitian structure $J$ of $\left(U, g_{*}\right)$ equals $\Omega_{*}=\theta_{1} \wedge \theta_{3}+\theta_{2} \wedge \theta_{4}$. Thus $\Omega_{*} \wedge \Phi=0$ and $J, \Phi^{\sharp}$ are orthogonal. Now it is clear that $d \Phi=0$, i.e. $\Phi^{\sharp}$ is an almost Kähler structure on $\left(U, g_{*}\right)$. It follows that (see $[\mathrm{S}]$ ) $\Psi^{\sharp}=J \circ \Phi^{\sharp}$ is also an almost Kähler structure on $\left(U, g_{*}\right)$. Note that $\Psi=$ $\theta_{1} \wedge \theta_{4}-\theta_{2} \wedge \theta_{3}$. Since $\xi v=\eta v=0$ it follows that $\xi, \eta$ are holomorphic Killing vector fields on $\left(U, g_{*}, J\right)$. Consequently, Lemma B implies that $\omega_{J V}=\theta_{3} \wedge \theta_{4}$ 
is a closed form. Thus the closed 2-form $\bar{\Phi}=\theta_{1} \wedge \theta_{2}-\theta_{3} \wedge \theta_{4}$ determines an opposite almost Kähler structure compatible with $g_{*}$. In view of Lemma B this form is an eigenvector of the negative Weyl tensor $W^{-}$of $\left(U, g_{*}\right)$. Since the distribution $J V$ is totally geodesic and preserved by the almost Kähler structure $\Phi^{\sharp}$ it follows that $\bar{\Phi}^{\sharp}$ is the natural opposite almost Hermitian structure of $\Phi^{\sharp}$. Since $(M, g, J)$ is conformally Kähler, $\Omega$ is an eigenvalue of the positive Weyl tensor $W^{+}$and $W^{+}$is degenerate. The eigenspace $L_{J} M$ corresponding to the eigenvalue of double multiplicity is clearly spanned by the forms $\Phi, \Psi$.

S. Salamon introduced in $[S$, p. 117, (c)] a class of compact complex 4-manifolds $(M, g)$ admitting a circle of almost Kähler structures $\Omega_{t}=$ $\cos t \Phi+\sin t \Psi$ where $g(\Phi, \Psi)=0$. It is clear that the form $\mu=\Phi+i \Psi$ is a holomorphic symplectic form trivializing the canonical bundle of $M$. Consequently, the Kodaira theorem ([Kod, Th. 19]) asserts that any such manifold is of the form $\mathbb{C}^{2} / \Gamma$ where $\Gamma$ is a group of affine transformations leaving invariant the standard symplectic form $d z^{1} \wedge d z^{2}$ (see [S], [Kod]). In $[\mathrm{S}]$ there is given an example of such manifolds called the Kodaira-Thurston manifold. Below we present other elementary examples on a standard 4-torus $T^{4}=\mathbb{C}^{2} / \Gamma$.

Corollary 4. On the torus $T^{4}$ there exist uncountably many Riemannian metrics $g_{\phi, \psi}$ admitting in both orientations a circle of almost Kähler structures which are eigenvalues of the Weyl tensor $W$ of $\left(T^{4}, g_{\phi, \psi}\right)$. The manifolds $\left(T^{4}, g_{\phi, \psi}\right)$ are not hyper-Kähler. These metrics are parameterized by smooth, real, periodic functions $\phi, \psi: \mathbb{R} \rightarrow \mathbb{R}$.

Proof. Consider the 2-tori $T^{2}=\mathbb{R}^{2} / 2 \pi \mathbb{Z}^{2}$ with metric

$$
g_{\phi}=d t^{2}+\exp 2 \phi(t) d s^{2}
$$

and standard complex structure $J_{\phi}$, where $\phi: \mathbb{R} \rightarrow \mathbb{R}$ is a smooth, periodic function satisfying $\phi(t+2 \pi)=\phi(t)$ and $J_{\phi}(\partial / \partial t)=\exp (-\phi) \partial / \partial s$. It is clear that $\left(T^{2}, g_{\phi}\right)$ admits in general only one Killing vector field $\xi_{\phi}=\partial / \partial s$. Note that on $T^{4}$ we have two complex structures of interest: $J=J_{\phi}+J_{\psi}$, $\bar{J}=J_{\phi}-J_{\psi}$, and both $\left(T^{4}, g, J\right)$ and $\left(T^{4}, g, \bar{J}\right)$, where $g=g_{\phi}+g_{\psi}$, are Kähler with two holomorphic Killing vector fields $\xi_{\phi}, \xi_{\psi}$. Now if $\left(T^{4}, g\right)=$ $\left(T^{2}, g_{\phi}\right) \times\left(T^{2}, g_{\psi}\right)$ then the Kähler surface $\left(T^{4}, g\right)$ (in both orientations) admits two holomorphic Killing vector fields $\xi_{\phi}, \xi_{\psi}$ such that $\left[\xi_{\phi}, \xi_{\psi}\right]=0$ and $\Omega\left(\xi_{\phi}, \xi_{\psi}\right)=0$. In our case the function $v=\exp (2 \phi+2 \psi)$ is nowhere zero on $M=T^{4}$. Consequently, the manifold $\left(T^{4}, g_{\phi, \psi}\right)$ with the metric $g_{\phi, \psi}=\exp -(\phi+\psi) g$ admits in both orientations two circles of almost Kähler structures. Every circle spans an eigenspace of the Weyl tensor $W^{ \pm}$ corresponding to the eigenvalue of $W^{ \pm}$of multiplicity 2 . 
Proposition 3. Let $(M, g, J)$ be a compact conformally Kähler Hermitian surface with $\chi(M) \neq 0$ whose group of holomorphic isometries contains a 2-torus. Then there exists an open and dense subset $U$ of $M$ such that $\left(U,\left.g\right|_{U}\right)$ is locally isometric to the manifold $\left(\mathbb{R}^{4}, g_{0}\right)$, where

$$
\begin{aligned}
g_{0}= & \phi\left(x_{1}, x_{2}\right) d x_{1}^{2}+2 \chi\left(x_{1}, x_{2}\right) d x_{1} \odot d x_{2}+\psi\left(x_{1}, x_{2}\right) d x_{2}^{2} \\
& +\phi\left(x_{1}, x_{2}\right) d x_{3}^{2}+2 \chi\left(x_{1}, x_{2}\right) d x_{3} \odot d x_{4}+\psi\left(x_{1}, x_{2}\right) d x_{4}^{2},
\end{aligned}
$$

$\phi(x)>0, \psi(x)>0, V(x)>0$ on $\mathbb{R}^{2}$ where $V(x)=\phi \psi-\chi^{2}$ and $\phi, \psi, \chi \in$ $C^{\infty}\left(\mathbb{R}^{2}\right)$. The complex structure on $\left(\mathbb{R}^{4}, g_{0}\right)$ is induced from $\mathbb{C}^{2}=\mathbb{R}^{4}$, explicitly $J\left(\partial / \partial x_{3}\right)=\partial / \partial x_{1}, J\left(\partial / \partial x_{4}\right)=\partial / \partial x_{2}$. The fields $\xi=\partial / \partial x_{3}, \eta=$ $\partial / \partial x_{4}$ are holomorphic vector fields on $\left(\mathbb{R}^{4}, J\right)$ and $g(\xi, \xi)=\phi, g(\xi, \eta)=\chi$, $g(\eta, \eta)=\psi$. The Kähler metric

$$
\begin{aligned}
g_{0}= & \frac{\partial^{2} f}{\partial x_{1}^{2}} d z_{1} \otimes d \bar{z}_{1}+\frac{\partial^{2} f}{\partial x_{1} \partial x_{2}} d z_{1} \otimes d \bar{z}_{2} \\
& +\frac{\partial^{2} f}{\partial x_{1} \partial x_{2}} d z_{2} \otimes d \bar{z}_{1}+\frac{\partial^{2} f}{\partial x_{2}^{2}} d z_{2} \otimes d \bar{z}_{2},
\end{aligned}
$$

where $f$ is some strictly convex function on $\mathbb{R}^{2}$, is toric.

Proof. See also [A]. Define $U=\left\{x \in M\right.$ : the $T^{2}$-action is free at $\left.x\right\}$ and let $V=g(\xi, \xi) g(\eta, \eta)-g(\xi, \eta)^{2}=\phi \psi-\chi^{2}$. Then $U=\{x: V(x) \neq 0\}$ and $\xi \neq 0, \eta \neq 0$ on $V$. Since the frame $\xi, \eta, J \xi, J \eta$ consists of Poisson commuting vector fields, we can find local coordinates on $U$ such that $\xi=\partial / \partial x_{3}$, $\eta=\partial / \partial x_{4}, J \xi=\partial / \partial x_{1}, J \eta=\partial / \partial x_{2}$. Now since $(M, g, J)$ is conformally Kähler, the conformal Kähler manifold is also toric. Assume that the metric $\bar{g}=F^{2} g_{0}$ is Kähler for some smooth function $F$. Note that $\theta=-2 d \ln F$, and so $F$ depends only on $x_{1}, x_{2}$. It is well known that if $\omega=i \sum g_{i \bar{j}} d z_{i} \wedge d \bar{z}_{j}$ is the Kähler form of a Kähler manifold $(M, \bar{g}, J)$ then locally $\omega=i \partial \bar{\partial} f$ for a certain real function $f$ defined locally on $M$. In our case

$$
g=\bar{\phi} d z_{1} \otimes d \bar{z}_{1}+\bar{\chi}\left(d z_{1} \otimes d \bar{z}_{2}+d z_{2} \otimes d \bar{z}_{1}\right)+\bar{\psi} d z_{2} \otimes d \bar{z}_{2}
$$

and

$$
\omega=i\left(\bar{\phi} d z_{1} \wedge d \bar{z}_{1}+\bar{\chi}\left(d z_{1} \wedge d \bar{z}_{2}+d z_{2} \wedge d \bar{z}_{1}\right)+\bar{\psi} d z_{2} \wedge d \bar{z}_{2}\right),
$$

where $z_{1}=x_{3}+i x_{1}, z_{2}=x_{4}+i x_{2}$ are local holomorphic coordinates on $M$. Since $\bar{\phi}, \bar{\chi}, \bar{\psi}$ depend only on $x_{1}, x_{2}$, to find examples of Kähler toric metrics we can consider the class of Kähler metrics for which $f=f\left(x_{1}, x_{2}\right)$, where we assume the function $\Phi\left(z_{1}, z_{2}\right)=f\left(x_{1}, x_{2}\right)$ to be strictly plurisubharmonic, which means that the matrix $\left(\partial^{2} \Phi / \partial z_{i} \partial \bar{z}_{j}\right)$ is positive definite, equivalently the matrix

$$
A=\left(\begin{array}{cc}
\partial^{2} f / \partial x_{1}^{2} & \partial^{2} f / \partial x_{1} \partial x_{2} \\
\partial^{2} f / \partial x_{1} \partial x_{2} & \partial^{2} f / \partial x_{2}^{2}
\end{array}\right)
$$


is positive definite. Now it is easy to check that such metrics are toric Kähler metrics with holomorphic Killing vector fields $\xi=\partial / \partial x_{3}, \eta=\partial / \partial x_{4}$. For such metrics

$$
\bar{\phi}=\frac{\partial^{2} f}{\partial x_{1}^{2}}, \quad \bar{\chi}=\frac{\partial^{2} f}{\partial x_{1} \partial x_{2}}, \quad \bar{\psi}=\frac{\partial^{2} f}{\partial x_{2}^{2}} .
$$

Corollary 5. Let $(M, g, J)$ be a Kähler toric surface. Then the Ricci form and scalar curvature $\tau$ of $(M, g, J)$ are determined by the volume function $V$ of the toric action and $\varrho=-i \partial \bar{\partial} \ln V, \tau=-\Delta \ln V$ in $U=\{x \in M$ : $V(x) \neq 0\}$. Assume that on $(M, J)$ there are given two toric Kähler metrics $g, g^{\prime}$ with the same Kähler class $\left(i . e .[\omega]=\left[\omega^{\prime}\right]\right)$ and with the same volume function of toric actions. If $M$ is compact then $g=g^{\prime}$.

Proof. The first part is obvious. The second is a consequence of the uniqueness part of the Calabi-Yau theorem (see [T-2]), since if $\varrho=\varrho^{\prime}$ on an open and dense subset of $M$ then $\varrho=\varrho^{\prime}$ on the whole of $M$.

Now we shall find conditions for the metric $g_{0}$ to be a toric Kähler metric which admits a negative almost Kähler structure constructed by the methods developed above. Note that the volume function $V$ of a toric action generated by the fields $\xi, \eta$ equals exactly $V=\operatorname{det} A=H(f)$ where $H(f)$ denotes the Hessian determinant of $f: U \rightarrow \mathbb{R}$ with respect to the Euclidean metric on $U \subset \mathbb{R}^{2}$. Thus we get

THEOREM 3. Assume that the function $\Phi\left(z_{1}, z_{2}\right)=f\left(x_{1}, x_{2}\right)$ is strictly plurisubharmonic on $M=\mathbb{R}^{2} \times i U$. Then the manifold $M$ with the metric

$$
\begin{aligned}
g= & \frac{1}{\sqrt{H(f)}}\left(\frac{\partial^{2} f}{\partial x_{1}^{2}} d z_{1} \otimes d \bar{z}_{1}+\frac{\partial^{2} f}{\partial x_{1} \partial x_{2}} d z_{1} \otimes d \bar{z}_{2}\right. \\
& \left.+\frac{\partial^{2} f}{\partial x_{1} \partial x_{2}} d z_{2} \otimes d \bar{z}_{1}+\frac{\partial^{2} f}{\partial x_{2}^{2}} d z_{2} \otimes d \bar{z}_{2}\right)
\end{aligned}
$$

admits in the positive orientation a circle of almost Kähler structures which are eigenvectors of the Weyl tensor $W^{+}$, and admits in the negative orientation an almost Kähler structure which is an eigenvector of the Weyl tensor $W^{-}$. If $H(f)$ is not constant then every almost Kähler structure from the circle is non-Kähler.

Proof. If there exists a (positive) Kähler structure on $(M, g, J)$ different from $J$ then $(M, g, J)$ is hyper-Kähler. Then $H(f)=$ const (see below).

Consequently, in that way we obtain a toric Kähler surface with an opposite almost Kähler structure if and only if $\operatorname{det} A=$ const, which means that $(M, g, J)$ is a Ricci flat Kähler surface, in particular, is an Einstein 4-manifold.

Summarizing we obtain a large class of Einstein almost Kähler metrics (in fact Ricci flat and hence hyper-Kähler) which we shall call Calabi metrics: 
THEOREM 4. Assume that $\Phi\left(z_{1}, z_{2}\right)=f\left(x_{1}, x_{2}\right)$ is a strictly plurisubharmonic function on $U \subset \mathbb{R}^{2}$. Assume that the function $f$ satisfies the real Monge-Ampère equation

$$
H(f)=\operatorname{det}\left(\begin{array}{cc}
\partial^{2} f / \partial x_{1}^{2} & \partial^{2} f / \partial x_{1} \partial x_{2} \\
\partial^{2} f / \partial x_{1} \partial x_{2} & \partial^{2} f / \partial x_{2}^{2}
\end{array}\right)=1 .
$$

Then the toric Kähler metric on the manifold $M=\mathbb{R}^{2} \times i U$ given by

$$
\omega=i\left(\frac{\partial^{2} f}{\partial x_{1}^{2}} d z_{1} \wedge d \bar{z}_{1}+\frac{\partial^{2} f}{\partial x_{1} \partial x_{2}}\left(d z_{1} \wedge d \bar{z}_{2}+d z_{2} \wedge d \bar{z}_{1}\right)+\frac{\partial^{2} f}{\partial x_{2}^{2}} d z_{2} \wedge d \bar{z}_{2}\right)
$$

is Ricci flat and admits (in the reversed orientation) an almost Kähler structure $I$ which is an eigenvalue of the Weyl tensor $W^{-}$of $(M, g, J)$. The structure $I$ is Kähler if and only if $f$ is a quadratic polynomial. The class of Calabi metrics is a subclass of the class of Gibbons-Hawking metrics.

Proof. The above metric coincides with the old Calabi construction ([Ca-1], [B, p. 423]). Note that $\omega^{2}=-H(f) d z_{1} \wedge d \bar{z}_{1} \wedge d z_{2} \wedge d \bar{z}_{2}$. Clearly the Ricci tensor of $(M, g, J)$ is $\varrho=i \partial \bar{\partial} \ln H(f)=0$. The holomorphic Killing vector fields are $\xi=\operatorname{Re} \partial / \partial z_{1}, \eta=\operatorname{Re} \partial / \partial z_{2}$. The volume function of the toric action defined by $\xi, \eta$ equals $H(f)=1$. Thus our theorem is a consequence of the results of Calabi and of Theorem 2 and Proposition 3. Since the almost Kähler structure $I$ of the above examples is an eigenvalue of the Weyl tensor $W^{-}$and clearly the Ricci tensor of $(M, g, J)$ is $I$-invariant it follows that Calabi metrics satisfy the Gray third symmetry condition $G_{3}$. The metrics satisfying this condition are classified in $[\mathrm{A}-\mathrm{A}-\mathrm{D}]$. If $\left(\Sigma, g_{\Sigma}\right)$ is a Riemannian surface with local isothermal coordinates $x, y$ and $h=w+i v$ is a holomorphic function on $\Sigma$ then the general $G_{3}$ almost Kähler metric on $\Sigma \times \mathbb{R}^{2}$ is

$$
g=g_{\Sigma}+w d z^{2}+\frac{1}{w}(d t+v d z)^{2} .
$$

The metric $g$ carries a Kähler structure with Kähler form $\bar{\omega}=\Omega_{\Sigma}+d z \wedge d t$. The Killing fields $K_{1}=\partial / \partial z, K_{1}=\partial / \partial t$ are holomorphic with respect to this Kähler structure, but $\bar{\omega}\left(K_{1}, K_{2}\right)=1$, i.e. the action is not toric. Note that the volume function $V=g\left(K_{1}, K_{1}\right) g\left(K_{2}, K_{2}\right)-g\left(K_{1}, K_{2}\right)^{2}$ of the action generated by $K_{1}=\partial / \partial z, K_{1}=\partial / \partial t$ on a general almost Kähler $G_{3}$ 4 -manifold is constant. Note that every Gibbons-Hawking metric is toric Kähler in our sense, with respect to a Kähler structure orthogonal to $J$, which is straightforward from their explicit construction. Note that the Calabi metrics cannot be complete and that always $U \neq \mathbb{R}^{2}$, which follows from the study of improper affine spheres ([Po], [Ca-2], [J-2]).

We shall finish with a certain local characterization of almost Kähler manifolds admitting an opposite (negative) almost Kähler structure. Our result is inspired by some well known results from foliation theory. 
We shall say that a point $x_{0} \in M$ is a point of positive sectional curvature of $(M, g)$ if $K(\pi)>0$ for all 2-planes $\pi \subset T_{x_{0}} M$ where $K$ denotes the sectional curvature of $(M, g)$. Let $P(M, g)=\{x: x$ is a point of positive sectional curvature of $(M, g)\}$. Then $P(M, g)$ is an open subset of $M$. We shall prove (see also [W, p. 16, Prop. 2.7]):

Theorem 5. Assume that $(M, g, J)$ is an almost Kähler 4-manifold admitting an opposite almost Kähler structure $\bar{J}$. Then $P(M, g)=\emptyset$. If the sectional curvature of $(M, g, J)$ is non-negative then $(M, g, J)$ is locally a product of two Riemannian surfaces with non-negative curvature and $J, \bar{J}$ are Kähler.

Proof. Let $\omega, \bar{\omega}$ be the Kähler forms of $(M, g, J),(M, g, \bar{J})$ respectively. Define $\omega_{1}=\frac{1}{2}(\omega+\bar{\omega}), \omega_{2}=\frac{1}{2}(\omega-\bar{\omega})$. Then $\omega_{i}^{2}=0$ and there exists a local frame $\left\{e_{1}, e_{2}, e_{3}, e_{4}\right\}$ such that $\omega_{1}=e_{1} \wedge e_{2}$ and $\omega_{2}=e_{3} \wedge e_{4}$. The forms $\omega_{i}$ define two minimal foliations as follows: $\mathcal{F}_{1}=\left\{X \in T M: i_{X} \omega_{2}=0\right\}=$ $\operatorname{span}\left\{e_{1}, e_{2}\right\}, \mathcal{F}_{2}=\left\{X \in T M: i_{X} \omega_{1}=0\right\}=\operatorname{span}\left\{e_{3}, e_{4}\right\}$. Now we shall use the Weitzenböck formulas to calculate $\left|\nabla \omega_{i}\right|^{2}$. Since $\Delta \omega=\Delta \bar{\omega}=0$ it follows that $\Delta \omega_{i}=0$. Thus

$$
0=\Delta \omega_{i}=\nabla^{*} \nabla \omega_{i}+\sum e_{j} \wedge\left(i_{e_{i}} R\left(e_{i}, e_{j}\right) \cdot \omega_{i}\right) .
$$

Now we compute $e_{j} \wedge\left(i_{e_{i}} R\left(e_{i}, e_{j}\right) . \omega\right)$ where $\omega=\omega_{1}$ :

$$
\begin{aligned}
e_{j} \wedge\left(i_{e_{i}} R\left(e_{i}, e_{j}\right) . \omega\right)= & \sum\left(e _ { j } \wedge \left(i_{e_{i}}\left(e_{1} \wedge e_{2}\right)\left(R\left(e_{i}, e_{j}\right) ., .\right)\right.\right. \\
& \left.\left.+i_{e_{i}}\left(e_{1} \wedge e_{2}\right)\left(., R\left(e_{i}, e_{j}\right) .\right)\right)\right) \\
= & \sum\left(e_{j} \wedge\left(g\left(R\left(e_{i}, e_{j}\right) e_{i}, e_{1}\right) e_{2}-g\left(R\left(e_{i}, e_{j}\right) e_{i}, e_{2}\right) e_{1}\right)\right. \\
& \left.+e_{j} \wedge\left(\delta_{1 i} g\left(R\left(e_{i}, e_{j}\right) e_{k}, e_{2}\right) e_{k}-\delta_{2 i} g\left(R\left(e_{i}, e_{j}\right) e_{i}, e_{1}\right) e_{k}\right)\right) \\
= & \sum\left(g\left(R\left(e_{i}, e_{j}\right) e_{k}, e_{2}\right) e_{j} \wedge e_{2}-g\left(R\left(e_{i}, e_{j}\right) e_{i}, e_{1}\right) e_{1} \wedge e_{j}\right) \\
& \left.+g\left(R\left(e_{1}, e_{j}\right) e_{k}, e_{2}\right) e_{j} \wedge e_{k}-g\left(R\left(e_{2}, e_{j}\right) e_{k}, e_{1}\right) e_{j} \wedge e_{k}\right) .
\end{aligned}
$$

On the other hand, since $g\left(\omega_{i}, \omega_{i}\right)=1$ we get $g\left(\nabla^{*} \nabla \omega_{i}, \omega_{i}\right)=g\left(\nabla \omega_{i}, \nabla \omega_{i}\right)$. Thus we obtain

$$
g\left(\nabla \omega_{1}, \nabla \omega_{1}\right)=-2 \sum_{i>2}\left(K\left(e_{i} \wedge e_{1}\right)+K\left(e_{i} \wedge e_{2}\right)\right) .
$$

Analogously we obtain

$$
g\left(\nabla \omega_{2}, \nabla \omega_{2}\right)=-2 \sum_{i<3}\left(K\left(e_{i} \wedge e_{3}\right)+K\left(e_{i} \wedge e_{4}\right)\right) .
$$

Thus $P(M, g)$ is empty. If the sectional curvature $K$ of $(M, g)$ is non-negative then $\nabla \omega_{1}=0, \nabla \omega_{2}=0$ and $(M, g)$ is locally a product of two (real) Riemannian surfaces with non-negative curvature and natural Kähler structures. 
EXAmple. The projective space $\mathbb{C} P^{2}$ with the Fubini-Studi metric does not admit any local opposite almost Kähler structure, although it admits plenty of local opposite complex orthogonal structures. The metrics (2.8) cannot have points of positive sectional curvature.

The paper was supported by KBN grant 2P0 3A 02324 . I am grateful to Prof. Ilka Agricola, Prof. Thomas Friedrich and the Institute of Mathematics of Humboldt University for warm hospitality.

\section{References}

[A] M. Abreu, Kähler geometry of toric manifolds in symplectic coordinates, arXiv DG/00041222v1 (2000).

[A-A-D] V. Apostolov, J. Armstrong and T. Draghici, Local models and integrability of certain almost Kähler 4-manifolds, Math. Ann. 323 (2002), 633-666.

[B] A. L. Besse, Einstein Manifolds, Springer, Berlin, 1987.

[Ca-1] E. Calabi, A construction of nonhomogeneous Einstein metrics, in: Differential Geometry, Proc. Sympos. Pure Math. 27, Amer. Math. Soc. 1975, 17-24.

[Ca-2] -, Improper affine hyperspheres of convex type and a generalization of a theorem by K. Jörgens, Michigan Math. J. 5 (1958), 105-126.

[J-1] W. Jelonek, Bihermitian Gray surfaces II, preprint, 2002.

[J-2] -, Solitons and projectively flat affine surfaces, in: D. Wójcik and J. Cieśliński (eds.), Nonlinearity and Geometry, PWN, Warszawa, 1998, 297-317.

[J-3] —, Einstein-Hermitian and anti-Hermitian 4-manifolds, Ann. Polon. Math. 81 (2003), 7-24.

[J-4] -, Bihermitian Gray surfaces, Pacific J. Math. 222 (2005), 57-68.

[Kob] S. Kobayashi, Transformation Groups in Differential Geometry, Springer, 1972.

[Kod] K. Kodaira, On the structure of compact complex analytic surfaces I, Amer. J. Math. 86 (1964), 751-798.

[L] C. Lebrun, Einstein metrics on complex surfaces, in: Geometry and Physics (Aarrhus, 1995), J. Anderson et al. (eds.), Lecture Notes in Pure and Appl. Math. 184, Dekker, 1997, 167-176.

[P] D. Page, A compact rotating gravitational instanton, Phys. Lett. B 79 B (1978), 235-238.

[Po] A. V. Pogorelov, On the improper convex affine hyperspheres, Geom. Dedicata 1 (1972), 33-46.

[S] S. Salamon, Special structures on four manifolds, Riv. Mat. Univ. Parma (4) 17 (1991), 109-123.

[T-1] G. Tian, On Calabi's conjecture for complex surfaces with positive first Chern class, Invent. Math. 101 (1990), 101-172.

[T-2] -, Canonical Metrics in Kähler Geometry, Lectures in Math. ETH Zürich, Birkhäuser, 2000.

[V] I. Vaisman, On locally and globally conformal Kähler manifolds, Trans. Amer. Math. Soc. 262 (1980), 533-542. 
[W] P. Walczak, On foliations with leaves satisfying some geometric conditions, Dissertationes Math. 226 (1983).

Institute of Mathematics

Technical University of Cracow

Warszawska 24

31-155 Kraków, Poland

E-mail: wjelon@pk.edu.pl

Received 22.9.2005

and in final form 8.1.2007

(1608) 